\title{
A RIEMANN-HILBERT PROBLEM AND THE BERNOULLI BOUNDARY CONDITION IN THE VARIATIONAL THEORY OF STOKES WAVES
}

\author{
E. SHARGORODSKY ${ }^{\mathrm{a}, *}$, J.F. TOLAND ${ }^{\mathrm{b}}$ \\ ${ }^{a}$ School of Mathematical Sciences, University of Sussex, Falmer, Brighton, BN1 9QH, UK \\ ${ }^{\mathrm{b}}$ Department of Mathematical Sciences, University of Bath, Claverton Down, Bath, BA2 7AY, UK
}

Received 4 September 2000, revised 26 June 2001, accepted 8 February 2002

ABSTRACT. - This paper concerns the question of equivalence between the Euler-Lagrange equation of a certain functional and periodic Stokes waves on the surface of an infinitely deep irrotational incompressible flow of an ideal fluid under gravity. Of particular concern is Bernoulli's constant-pressure condition on a free surface.

(C) 2003 L'Association Publications de l'Institut Henri Poincaré. Published by Elsevier B.V. All rights reserved

MSC: 35R35; 76B15; 44A15; 42A50

Keywords: Regularity theory; Free-boundary problem; Stokes waves; Riemann-Hilbert problem; Bernoulli problem

RÉSUMÉ. - Cet article concerne la question de l'équivalence entre les solutions de l'équation d'Euler-Lagrange pour une certaine fonctionnelle et les ondes périodiques de Stokes à la surface d'un liquide de profondeur infinie. Une attention particulière est portée sur la condition de Bernoulli qui dit que le pression est atmosphérique le long de la frontière libre.

(C) 2003 L'Association Publications de l'Institut Henri Poincaré. Published by Elsevier B.V. All rights reserved

\section{Introduction and main results}

A Stokes wave is a steady periodic wave, propagating under gravity with constant speed $c$ on the surface of an infinitely deep irrotational flow. Its free surface is determined by Laplace's equation, kinematic and periodic boundary conditions and by a dynamic boundary condition given by the requirement that pressure in the flow at the surface should be constant (Bernoulli's theorem). Recently [2,3] Stokes waves have been seen

\footnotetext{
* Corresponding author.

E-mail addresses: e.shargorodsky@ sussex.ac.uk (E. Shargorodsky), jft@ maths.bath.ac.uk (J.F. Toland).
} 
to arise from critical points of the functional

$$
\mathcal{J}(w)=\int_{-\pi}^{\pi}\left\{w \mathcal{C} w^{\prime}-\lambda w^{2}\left(1+\mathcal{C} w^{\prime}\right)\right\} \mathrm{d} x
$$

$w \in W_{2 \pi}^{1,2}$ and $\lambda>0$. Here $\mathcal{C} u$ denotes the periodic Hilbert transform of a $2 \pi$-periodic function $u: \mathbb{R} \rightarrow \mathbb{R}:$

$$
\mathcal{C} u(x)=\frac{1}{2 \pi} \int_{-\pi}^{\pi} u(y) \cot \left(\frac{1}{2}(x-y)\right) \mathrm{d} y,
$$

see $[17,18]$. For $p \geqslant 1, W_{2 \pi}^{1, p}$ is the linear space of absolutely continuous, $2 \pi$-periodic functions $w$ with weak first derivatives $w^{\prime} \in L_{2 \pi}^{p}$ and, for $p>0, L_{2 \pi}^{p}$ denotes the linear space of $2 \pi$-periodic locally $p$ th-power summable functions. The Euler-Lagrange equation, to be satisfied by critical points of $\mathcal{J}$, is

$$
\mathcal{C} w^{\prime}=\lambda\left\{w+w \mathcal{C} w^{\prime}+\mathcal{C}\left(w w^{\prime}\right)\right\}, \quad \lambda>0 .
$$

A formal derivation of this variational principle for Stokes waves appeared in [4], independently of the earlier work by Babenko [1] and Plotnikov [12]. Most recently [15] critical points of $\mathcal{J}$ have been studied in $W_{2 \pi}^{1, p}, 1<p<2$, and in the real Hardy space $\mathcal{H}_{\mathbb{R}}^{1,1}$ of absolutely continuous periodic functions with derivative in the usual Hardy space $\mathcal{H}_{\mathbb{R}}^{1}:=\left\{u \in L_{2 \pi}^{1}: \mathcal{C} u \in L_{2 \pi}^{1}\right\}$. (Note that $W_{2 \pi}^{1, p} \subset \mathcal{H}_{\mathbb{R}}^{1,1}$ for $p>1$.) The conclusion was the following.

THEOREM 1.1. - For solutions $w \in \mathcal{H}_{\mathbb{R}}^{1,1}$ of (1.2) the following statements are equivalent:

$$
\begin{aligned}
& 1-2 \lambda w \geqslant 0 \text { almost everywhere; } \\
& (1-2 \lambda w)\left\{w^{\prime 2}+\left(1+\mathcal{C} w^{\prime}\right)^{2}\right\}=1 \text { almost everywhere; } \\
& \int_{-\pi}^{\pi}|1-2 \lambda w|\left\{\left(w^{\prime}\right)^{2}+\left(\mathcal{C} w^{\prime}\right)^{2}\right\} \mathrm{d} x<\infty .
\end{aligned}
$$

In these circumstances $w$ is real-analytic on the open set where $1-2 \lambda w>0$. Moreover this is best possible, in the sense that there is a solution $(\hat{\lambda}, \hat{w}) \in(0, \infty) \times\left\{\bigcap_{p<3} W_{2 \pi}^{1, p}\right\}$ with $1-2 \hat{\lambda} \hat{w}>0$ almost everywhere but which is is not Lipschitz continuous at a discrete set of points where $1-2 \hat{\lambda} \hat{w}=0$.

The significance of this result derives from the fact (see [2, (2.2) and the proof of Theorem 2.3]) that $(\beta)$ is equivalent to the Bernoulli constant-pressure condition for a Stokes wave with Froude number $1 / \sqrt{\lambda}$ in the following sense. If $(\beta)$ holds and the periodic profile, given in dimensionless coordinates, by

$$
\mathcal{S}:=\{(-t-\mathcal{C} w(t), w(t)): t \in \mathbb{R}\}
$$


is non-self-intersecting, then $w$ gives rise to a Stokes wave. In other words, $(\beta)$ then implies the existence of a complex analytic function $\varphi+\mathrm{i} \psi$ on the region below $\mathcal{S}$ in the complex plane which satisfies the Bernoulli condition

$$
\frac{1}{2}|\nabla \varphi(x, y)|^{2}+\lambda y \equiv \frac{1}{2} \quad \text { and } \quad \psi(x, y)=0 \quad \text { for all }(x, y) \in \mathcal{S} .
$$

Here, as usual, $\varphi$ is the velocity potential and $\psi$ is the stream function in dimensionless coordinates. For an actual wave $\lambda=g \Lambda / \pi c^{2}$, where $\Lambda$ is the wavelength, $c$ the velocity and $g$ is gravity. For the solution $(\hat{\lambda}, \hat{w})$ mentioned in Theorem 1.1, (1.3) gives the profile $\widehat{\mathcal{S}}$ of a Stokes wave of extreme form which has a corner at its highest point. The question arises as to whether it can be established that $(\beta)$ is satisfied by all solutions of (1.2). To describe progress so far we need to review the proof of Theorem 1.1. The key step is a reduction of (1.2) to a function-theoretic question on the unit disc $D$ centred at 0 in the complex plane. The following background material is from $[6,7,13,18]$.

For a holomorphic function $f: D \rightarrow \mathbb{C}$, let $f_{r}(t)=f\left(r \mathrm{e}^{\mathrm{i} t}\right)$ for $t \in \mathbb{R}$ and $r \in(0,1)$. Then, for any $p \in(0, \infty]$,

$$
\|f\|_{p}=\lim _{r \rightarrow 1}\left\|\left|f_{r}\right|\right\|_{L_{2 \pi}^{p}}=\sup _{r \in(0,1)}\left\|\left|f_{r}\right|\right\|_{L_{2 \pi}^{p}}
$$

is well defined. The Hardy class $\mathcal{H}_{\mathbb{C}}^{p}$ is the set of all such functions $f$ with $\|f\|_{p}<\infty$. Moreover for any $f \in \mathcal{H}_{\mathbb{C}}^{p}, p \in(0, \infty], f^{*}(t)=\lim _{r \rightarrow 1} f_{r}(t)$ is well defined for almost all $t \in \mathbb{R},\left|f^{*}\right| \in L_{2 \pi}^{p},\left\|\left|f^{*}\right|\right\|_{L_{2 \pi}^{p}}=\|f\|_{p}$ and $\log \left|f^{*}\right| \in L_{2 \pi}^{1}$ if $f \not \equiv 0$.

THEOREM 1.2 (Smirnov). - Suppose that $F \in \mathcal{H}_{\mathbb{C}}^{p}, p>0$, and that $F^{*} \in L_{2 \pi}^{q}, q>p$. Then $F \in \mathcal{H}_{\mathbb{C}}^{q}$.

The space $\mathcal{H}_{\mathbb{R}}^{1,1}$ is a Banach algebra (see [15] for an elementary proof) and $u \in \mathcal{H}_{\mathbb{R}}^{1}$ if and only if $u+\mathrm{i} \mathcal{C} u=U^{*}$ for some $U \in \mathcal{H}_{\mathbb{C}}^{1}$.

We can rewrite (1.2) for a function $w \in \mathcal{H}_{\mathbb{R}}^{1,1}$ as follows. Let $u=(1-2 \lambda w)\left(1+\mathcal{C} w^{\prime}\right)$. It then follows from $(1.2)$ that $u=1-\mathcal{C}\left((1-2 \lambda w) w^{\prime}\right)$ and $u \in \mathcal{H}_{\mathbb{R}}^{1}$. In other words

$$
u+\mathrm{i} \mathcal{C} u=(1-2 \lambda w)\left\{1+\mathcal{C} w^{\prime}+\mathrm{i} w^{\prime}\right\}
$$

Let $W, U \in \mathcal{H}_{\mathbb{C}}^{1}$ be such that $U^{*}=-\mathrm{i}(u+\mathrm{i} \mathcal{C} u)$ and $W^{*}=w^{\prime}+\mathrm{i}\left(1+\mathcal{C} w^{\prime}\right)$. Then (1.2) is equivalent to the following nonlinear Riemann-Hilbert problem

$$
U^{*}=(1-2 \lambda w) \overline{W^{*}} .
$$

The following theorem [7], due to Carleman, and an outer function construction [13], were crucial in the proof of Theorem 1.1 and will be used in the sequel.

THEOREM 1.3 (Carleman). - Suppose that $F, G \in \mathcal{H}_{\mathbb{C}}^{1}$ and that $F^{*}=\overline{G^{*}}$ almost everywhere on an open segment $\Gamma$ of $\partial D$. Then $F$ has an analytic continuation $\hat{F}: D \cup \Gamma \cup(\mathbb{C} \backslash \bar{D}) \rightarrow \mathbb{C}$ given by $\hat{F}(z)=\overline{G(1 / \bar{z})}, z \in \Gamma \cup(\mathbb{C} \backslash \bar{D})$. Similarly for $G$. In particular, if $\Gamma=\partial D$ then $F$ and $G$ are constant functions. 
For a $2 \pi$-periodic function $g: \mathbb{R} \rightarrow \mathbb{C}$ such that $\log |g| \in L_{2 \pi}^{1}$, an outer function $\mathcal{O}(g)$ is defined by

$$
\mathcal{O}(g)(z)=\exp \left\{\frac{1}{2 \pi} \int_{-\pi}^{\pi} \frac{\mathrm{e}^{\mathrm{i} t}+z}{\mathrm{e}^{\mathrm{i} t}-z} \log |g|(t) \mathrm{d} t\right\}, \quad z \in D .
$$

Outer functions have the following properties [13, Theorems 17.16 and 17.7]:

(A) $\mathcal{O}(g)$ is holomorphic, has no zeros in $D$ and $\mathcal{O}(g)(0) \in \mathbb{R}$;

(B) for $g_{1}, g_{2}$ with $\log \left|g_{1}\right|, \log \left|g_{2}\right| \in L_{2 \pi}^{1}, \mathcal{O}\left(g_{1} g_{2}\right)=\mathcal{O}\left(g_{1}\right) \mathcal{O}\left(g_{2}\right)$;

(C) for $g$ with $\log |g| \in L_{2 \pi}^{1}$ and $p \in(0, \infty],|g| \in L_{2 \pi}^{p}$ if and only if $\mathcal{O}(g) \in \mathcal{H}_{\mathbb{C}}^{p}$, and then $\left|(\mathcal{O}(g))^{*}\right|=|g|$;

(D) for $f \in \mathcal{H}_{\mathbb{C}}^{p}, p \in(0, \infty]$ and $z \in D,\left|\mathcal{O}\left(f^{*}\right)(z)\right| \geqslant|f(z)|$.

A consequence of the following corollary of Carleman's theorem is a small extension of part of Theorem 1.1.

Lemma 1.4. - Suppose that $\Phi, \Psi \in \mathcal{H}_{\mathbb{C}}^{1}$ are such that

$$
\Psi^{*}=a \overline{\Phi^{*}}
$$

and $a\left|\Phi^{*}\right|^{2}$ is bounded below, where $a$ is a real-valued function on $[-\pi, \pi]$. Then $\Phi \Psi$ is constant on $D$ and $a\left|\Phi^{*}\right|^{2}=c$ almost everywhere, where $c$ is a constant. (In particular, $a$ is either positive or negative on a set of full measure if $\Psi \not \equiv 0$.)

Proof. - Suppose $(\Phi, \Psi) \in \mathcal{H}_{\mathbb{C}}^{1} \times \mathcal{H}_{\mathbb{C}}^{1}$ and $a$ are as in the statement. Then

$$
\Phi^{*} \Psi^{*}=a\left|\Phi^{*}\right|^{2} \geqslant-d, \quad d>0 \text { a constant }
$$

and $f^{*} \geqslant 1$, where $f:=\Phi \Psi+d+1 \in \mathcal{H}_{\mathbb{C}}^{1 / 2}$. Therefore by a result, proved independently by Helson and Sarason and by Neuwirth and Newman, $f$ is constant on $D$ (see [6, Chapter II, Exercise 13]). There follows a short proof of this fact, based on the theorems of Carleman and Smirnov, which is in the spirit of the rest of this paper. Note that

$$
F^{*}=\overline{G^{*}}, \quad \text { where } F=\frac{f}{\mathcal{O}\left(\sqrt{f^{*}}\right)}, G=\mathcal{O}\left(\sqrt{f^{*}}\right),
$$

and both sides of this equation are in $H_{\mathbb{C}}^{1}$ (by Smirnov's theorem, since the right side is). Therefore, by Carleman's theorem $\mathcal{O}\left(\sqrt{f^{*}}\right)$, and hence $f$, is constant on $D$. Therefore $\Phi \Psi$ is constant on $D$. The result now follows.

COROLLARY 1.5. - This shows that in the first theorem $(\alpha)-(\gamma)$ is a consequence of the hypothesis

$$
(1-2 \lambda w)\left\{w^{\prime 2}+\left(1+\mathcal{C} w^{\prime}\right)^{2}\right\} \text { is bounded below, }
$$

which is weaker than $(\alpha),(\beta)$.

For the next result, which is a further refinement of Theorem 1.1, we need a definition. 
DEFINITION 1.6. - For any continuous function a which is non-zero almost everywhere on $[-\pi, \pi]$, let $\Sigma(a)=\operatorname{sgn}\{a\}$. Note that $\Sigma(a)$ is constant on each of an at most countable family $\mathcal{G}$ of open segments $\Gamma$ of $\mathbb{R}$, the union of which has full measure. Consider the set

$$
\mathcal{P}(a)=\left\{(\varphi, \psi) \in \mathcal{H}_{\mathbb{C}}^{1} \times \mathcal{H}_{\mathbb{C}}^{1}: \psi^{*}=\Sigma(a) \overline{\varphi^{*}} \text { almost everywhere on } \mathbb{R}\right\},
$$

and note from Carleman's theorem that both $\varphi$ and $\psi$ are analytic at every point of $\left\{\mathrm{e}^{\mathrm{i} t} \mid t \in \Gamma\right\}$ for all $\Gamma \in \mathcal{G}$. Note also that $\varphi^{*} \psi^{*}=\Sigma(a)\left|\varphi^{*}\right|^{2}$ is real-valued on $\mathbb{R}$. Now define the set $\mathcal{Z}(a)$ by

$$
\mathcal{Z}(a)=\left\{\varphi^{*} \psi^{*}:(\varphi, \psi) \in \mathcal{P}(a)\right\}
$$

Then each $z \in \mathcal{Z}(a)$ is real-valued almost everywhere on $\mathbb{R}$ and is real-analytic on $\bigcup_{\Gamma \in \mathcal{G}} \Gamma$. Note also that on $\Gamma \in \mathcal{G}$, any function $z \in \mathcal{Z}(a)$ has the same sign as $a$.

Remark. $-\mathcal{Z}(a)$, for any continuous function $a$ which is non-zero almost everywhere, is a family of functions on $[-\pi, \pi]$ determined solely by the set $Z(a)$ of points where $a$ is negative on $[-\pi, \pi]$. See Section 4 for further remarks on $\mathcal{Z}$.

THEOREM 1.7. - Suppose that $(\lambda, w) \in(0, \infty) \times \mathcal{H}_{\mathbb{R}}^{1,1}$ is a solution of $(1.2)$.

(a) The following are equivalent: (i) $w \in W_{2 \pi}^{1,3}$; (ii) $w$ is real-analytic on $\mathbb{R}$; (iii) 1 $2 \lambda w>0$ on $\mathbb{R}$.

(b) If $w \in W_{2 \pi}^{1,3 / 2}$ then $(\alpha)-(\gamma)$ hold.

(c) If $w \in W_{2 \pi}^{1, p}, 1 \leqslant p<3 / 2$, then $w$ is real-analytic on the open set of full measure where $1-2 \lambda w \neq 0$ and

$$
(1-2 \lambda w(t))\left\{w^{\prime}(t)^{2}+\left(1+\mathcal{C} w^{\prime}(t)\right)^{2}\right\}=z(t) \in \mathcal{Z}(1-2 \lambda w)
$$

(d) Suppose that $(\alpha)$ holds and

$$
\left(1+\mathcal{C} w^{\prime}\right)+\mathrm{i} w^{\prime}=\left|\left(1+\mathcal{C} w^{\prime}\right)+\mathrm{i} w^{\prime}\right| \mathrm{e}^{\mathrm{i} \vartheta}
$$

If $\vartheta=\vartheta_{1}+\vartheta_{2}$, where $\vartheta_{1}$ is continuous and $\left\|\vartheta_{2}\right\|_{\infty}<\pi / 6$, then $w$ is real-analytic.

Proof. - The proof is given in Section 3.

Remark. - We have seen that condition $(\beta)$ in Theorem 1.1 is sufficient to ensure that a solution of (1.2) corresponds to a Stokes waves in classical hydrodynamics, provided that the corresponding profile (1.3) is a non-self-intersecting curve. (Indeed we know for smooth solutions, and for solutions that arise as limits of smooth solutions, that $(\beta)$ in Theorem 1.1 is sufficient to ensure that the profile is non-self-intersecting. For these solutions there is nothing more to prove [16].) If however $(\delta)$ holds and $z \not \equiv 1$, the freeboundary condition (1.4) for $\varphi+\mathrm{i} \psi$ becomes

$$
\frac{1}{2} z(\varphi(x, y))|\nabla \varphi(x, y)|^{2}+\lambda y \equiv \frac{1}{2} \quad \text { and } \quad \psi(x, y)=0 \quad \text { for all }(x, y) \in \mathcal{S}
$$

which is not a constant-pressure condition on the free surface $\mathcal{S}$. 
Part (a) is a strong reminder of the intimate connection between the positivity of $1-2 \lambda w$ in Eq. (1.2) and the regularity of $w$. Also $(\hat{\lambda}, \hat{w})$ in Theorem 1.1 shows the sense in which $p=3$ in part (a) is sharp. In that example $\vartheta$ is continuous everywhere on $[-\pi, \pi]$ except at 0 where it jumps by $\pi / 3$. It can, therefore, be represented in the form $\vartheta=\vartheta_{1}+\vartheta_{2}$, where $\vartheta_{1}$ is continuous and $\left\|\vartheta_{2}\right\|_{\infty}=\pi / 6$, but $\left\|\vartheta_{2}\right\|_{\infty}$ cannot be made less than $\pi / 6$, so part (d) is sharp. (There are however real-analytic solutions with $\sup |\vartheta|>\pi / 6$, see [10].) Part (b) shows how Bernoulli's condition follows if $w \in W_{2 \pi}^{1,3 / 2}$.

A question remains: "are there solutions of (1.2) for which the Bernoulli condition $(\beta)$ fails?" In part (c) it is shown that, although we are unable to settle this basic question, in all cases the solutions $w \in \mathcal{H}_{\mathbb{R}}^{1,1}$ of (1.2) satisfy a generalised Bernoulli condition. This is considered in Section 4, where it is shown that the generalised Bernoulli condition is the best that can be predicted by the linear theory which is developed in Section 2 . Therefore, if the true Bernoulli condition (1.4) holds, it must be a further consequence of (1.2). In Section 4 we examine this condition when $1-2 \lambda w$ changes sign finitely often and we observe its particularly explicit form when $1-2 \lambda w$ changes sign exactly twice.

\section{Basic Riemann-Hilbert theory}

Before we go any further we record a result the proof of which is identical to that of Theorem 1.1 (see [15]) and Lemma 1.4.

Proposition 2.1. - Suppose that $\Phi, \Psi \in \mathcal{H}_{\mathbb{C}}^{1}, \Phi \not \equiv 0, a \in L_{2 \pi}^{\infty}$ is real-valued and $\Psi^{*}=a \overline{\Phi^{*}}$. Then the following are equivalent.

(a) a has the same sign almost everywhere.

(b) $a\left|\Phi^{*}\right|^{2}=$ const almost everywhere.

(c) $|a|\left|\Phi^{*}\right|^{2} \in L_{2 \pi}^{1}$.

(d) $a\left|\Phi^{*}\right|^{2}$ is bounded above (or below).

Proof. - Since the fact that (c) implies (b) is relevant in the proof of Theorem 2.2, we sketch a proof in the present notation. For the rest, see [15]. Suppose (c) and (2.1) hold and that $\Psi \neq \equiv 0$. (If $\Psi \equiv 0$ the result is trivial.) Then $\Psi^{2^{*}}=a^{2} \overline{\Phi^{2^{*}}}$ and, by the remarks before Smirnov's Theorem 1.2, $\log |a| \in L_{2 \pi}^{1}$. Let

$$
K=\mathcal{O}(a), \quad F(z)=\frac{\Psi^{2}(z)}{K(z)} \quad \text { and } \quad G(z)=K(z) \Phi^{2}(z), \quad z \in D
$$

Then $F^{*}=\overline{G^{*}}$, and from the properties (A)-(D) of an outer function we find that

$$
|F(z)|,|G(z)| \leqslant\left|\mathcal{O}\left(G^{*}\right)(z)\right|, \quad z \in D
$$

(cf. the proof of Theorem 1.7(c)). Since $G^{*} \in L_{2 \pi}^{1}$ by hypothesis, we find that $F, G \in$ $\mathcal{H}_{\mathbb{C}}^{1}$. Thus both are constants. Hence $(\Psi \Phi)^{2}=F G=$ constant. Therefore $\Psi \Phi$ is a constant on $D$, and (b) follows.

Let $C_{2 \pi}^{\alpha}$ denote the space of $2 \pi$-periodic functions on $\mathbb{R}$ which are Hölder continuous with exponent $\alpha \in(0,1]$, where $\alpha=1$ means Lipschitz continuity. Let $a \in C_{2 \pi}^{\alpha}$, and 
consider the problem

$$
\Psi^{*}=a \overline{\Phi^{*}}, \quad \text { where } \Phi, \Psi \in \mathcal{H}_{\mathbb{C}}^{p}, 1 \leqslant p \leqslant \infty
$$

THEOREM 2.2. - Suppose a changes sign. Then (2.1) does not have non-trivial solutions if

$$
p \geqslant \frac{2}{1+\alpha}
$$

Remark. - The example in the remark following Lemma 4.2 shows that Theorem 2.2 is sharp.

Proof. - Let $\xi, \eta \in L_{2 \pi}^{p}$ be such that

$$
\Psi^{*}=\xi+\mathrm{i}(c+\mathcal{C} \xi) \text { and } \Phi^{*}=\eta+\mathrm{i}(d+\mathcal{C} \eta),
$$

where $c, d$ are real constants. Then

$$
\xi=a \eta \quad \text { and } \quad c+\mathcal{C} \xi=-a(d+\mathcal{C} \eta) .
$$

Hence

$$
\mathcal{C}(a \eta)+a \mathcal{C} \eta+c+d a \equiv 0 .
$$

Define $\mathcal{Q}_{a}(\eta)$ by $\mathcal{Q}_{a}(\eta)(t)=a(t) \mathcal{C} \eta(t)-\mathcal{C}(a \eta)(t), t \in[-\pi, \pi]$. Then, by periodicity,

$$
\begin{aligned}
\left|\mathcal{Q}_{a}(\eta)(t)\right| & =\left|\frac{1}{2 \pi} \int_{t-\pi}^{t+\pi} \frac{(a(t)-a(s)) \eta(s)}{\tan \frac{1}{2}(t-s)} \mathrm{d} s\right| \leqslant \frac{\|a\|_{C_{2 \pi}^{\alpha}}}{\pi} \int_{t-\pi}^{t+\pi} \frac{|\eta(s)|}{\left|2 \tan \frac{1}{2}(t-s)\right|^{1-\alpha}} \mathrm{d} s \\
& \leqslant \frac{\|a\|_{C_{2 \pi}^{\alpha}}}{\pi} \int_{t-\pi}^{t+\pi} \frac{|\eta(s)|}{|t-s|^{1-\alpha}} \mathrm{d} s \leqslant \frac{\|a\|_{C_{2 \pi}^{\alpha}}}{\pi} \int_{-2 \pi}^{2 \pi} \frac{|\eta(s)|}{|t-s|^{1-\alpha}} \mathrm{d} s .
\end{aligned}
$$

Since $\eta \in L_{2 \pi}^{p} \subset L_{2 \pi}^{2 /(1+\alpha)}$, the Hardy-Littlewood-Sobolev inequality [9, p. 98] gives that the right hand side is in $L^{2 /(1-\alpha)}(\mathbb{R})$, and therefore $\mathcal{Q}_{a}(\eta)$ is in $L_{2 \pi}^{2 /(1-\alpha)}$. Since

$$
2 a \mathcal{C} \eta=\mathcal{Q}_{a}(\eta)-c-d a \text { and } 2 \mathcal{C}(a \eta)=-\mathcal{Q}_{a}(\eta)-c-d a
$$

we find that $a \mathcal{C} \eta, \mathcal{C}(a \eta) \in L_{2 \pi}^{2 /(1-\alpha)}$. Hence, by the Riesz theorem, $a \eta \in L_{2 \pi}^{2 /(1-\alpha)}$, and it follows from Hölder's inequality that $\Psi^{*} \Phi^{*}=a\left\{\eta^{2}+(d+\mathcal{C} \eta)^{2}\right) \in L_{2 \pi}^{1}$. However, this means that $|a|\left|\Phi^{*}\right|^{2} \in L_{2 \pi}^{1}$ and hence $a$ does not change sign, by Proposition 2.1.

COROllary 2.3. - Suppose $a \neq \equiv$ and $a\left(t_{0}\right)=0$ for some $t_{0} \in[0,2 \pi]$. Then (a) (2.1) does not have non-trivial solutions if $p \geqslant 2 / \alpha$, and (b) if $a^{-1} \notin L_{2 \pi}^{1}$ and $p \geqslant 2$, (2.1) does not have non-trivial solutions.

Proof. - Because of Theorem 2.2 we only need to consider the case when $a$ does not change sign. Multiplying $\Phi$ by -1 if necessary, we can assume that $a \geqslant 0$. As in the proof of Proposition 2.1, $\log a \in L_{2 \pi}^{1}$. Suppose that $(\Phi, \Psi) \in \mathcal{H}_{\mathbb{C}}^{p} \times \mathcal{H}_{\mathbb{C}}^{p}$ is a non-trivial 
solution of (2.1) and let $F=\Psi / H$ and $G=H \Phi$, where $H=\mathcal{O}(\sqrt{a})$. From properties (A)-(D) of outer functions it follows, as in the proof of Theorem 1.1, Proposition 2.1 and Theorem 1.7(c) below, that $F, G \in \mathcal{H}_{\mathbb{C}}^{p}, F^{*}=\overline{G^{*}}$ and hence $G \equiv b=$ const. Since $\Phi \neq 0, b \neq 0$. We have $\Phi=b H^{-1}$. Therefore

$$
\left|\Phi^{*}(t)\right|=\mathrm{const}|\sqrt{a(t)}|^{-1} \geqslant \mathrm{const}\left|t-t_{0}\right|^{-\alpha / 2}>0
$$

Thus $\Phi$ cannot belong to $\mathcal{H}_{\mathbb{C}}^{p}$ if $p \geqslant 2 / \alpha$. This proves (a). It follows from the equality $\left|\Phi^{*}\right|^{2}=|b|^{2} a^{-1}$ that if $a^{-1} \notin L_{2 \pi}^{1}$ then $\Phi^{*} \notin L_{2 \pi}^{2}$, which proves (b).

\section{Proof of Theorem 1.7}

LEMMA 3.1. - Let $(\lambda, w) \in(0, \infty) \times \mathcal{H}_{\mathbb{R}}^{1,1}$ be a solution of $(1.2)$. Then $\log \mid 1-$ $2 \lambda w \mid \in L_{2 \pi}^{1}$ and $1-2 \lambda w>0$ on a set of positive measure.

Proof. - Let $W, U \in \mathcal{H}_{\mathbb{C}}^{1}$ be defined following (1.5) so that Eq. (1.6) holds. It follows from $W^{*}=w^{\prime}+\mathrm{i}\left(1+\mathcal{C} w^{\prime}\right)$ and $U^{*}=-\mathrm{i}(u+\mathrm{i} \mathcal{C} u), u=1-\mathcal{C}\left((1-2 \lambda w) w^{\prime}\right)$ that

$$
W(0)=\mathrm{i}, \quad U(0)=-\mathrm{i} .
$$

Hence $W, U \neq \equiv 0$ and it follows from (1.6) that $\log |1-2 \lambda w|=\log \left|U^{*}\right|-\log \left|W^{*}\right| \in$ $L_{2 \pi}^{1}$.

An integration of (1.2) gives that

$$
\int_{-\pi}^{\pi} w \mathrm{~d} t=-\int_{-\pi}^{\pi} w \mathcal{C} w^{\prime} \mathrm{d} t=-2 \pi \sum_{k \in \mathbb{Z}}|k|\left|a_{k}\right|^{2}<0,
$$

where the $a_{k}$ are the Fourier coefficients of $w$. This implies that $w<0$ on a set of positive measure. So, $1-2 \lambda w>0$ on a set of positive measure.

(a) If $1-2 \lambda w>0$ on $\mathbb{R}$ then $(\alpha)-(\gamma)$ hold and it follows that $w \in W_{2 \pi}^{1,2}$. Therefore, by the result of [2], $w$ is real-analytic on $\mathbb{R}$. Thus (iii) implies (i) and (ii). Now suppose that (i) holds. Then $1-2 \lambda w \in C_{2 \pi}^{2 / 3}$, by Hölder's inequality, and $U, W \in \mathcal{H}_{\mathbb{C}}^{3}$ in (1.6). Corollary 2.3 implies that $1-2 \lambda w$ is nowhere zero, i.e. $1-2 \lambda w>0$ on $\mathbb{R}$ (by Lemma 3.1). This proves that (i) implies (iii) and (ii). Since (ii) implies (i) the proof is complete.

(b) By Theorem 1.1 it will suffice to show that $1-2 \lambda w>0$ almost everywhere. Now $w \in C_{2 \pi}^{1 / 3}$ and $U, W \in \mathcal{H}_{2 \pi}^{3 / 2}$ in (1.6), since $w^{\prime} \in L_{2 \pi}^{3 / 2}$, and it is immediate from Theorem 2.2 that $1-2 \lambda w$ does not change sign, i.e. $1-2 \lambda w>0$ almost everywhere (by Lemma 3.1).

(c) The continuous function $1-2 \lambda w$ is non-zero on a set of full measure (see Lemma 3.1). Let $\sigma=\Sigma(1-2 \lambda w)$ and let $H=\mathcal{O}(\sqrt{|1-2 \lambda w|})$. Then

$$
\left(\frac{U}{H}\right)^{*}=\sigma \overline{(H W)^{*}}
$$


Note that

$$
\begin{aligned}
\left|\frac{U(z)}{H(z)}\right| & \leqslant\left|\frac{\mathcal{O}\left(U^{*}\right)(z)}{\mathcal{O}(\sqrt{|1-2 \lambda w|})(z)}\right|=\left|\mathcal{O}\left(\frac{U^{*}}{H^{*}}\right)(z)\right|=\left|\mathcal{O}\left(H^{*} W^{*}\right)(z)\right| \\
& =\left|\mathcal{O}\left(W^{*}\right)(z)\right||\mathcal{O}(\sqrt{|1-2 \lambda w|})(z)|
\end{aligned}
$$

and $\left|\mathcal{O}(\sqrt{|1-2 \lambda w|})^{*} W^{*}\right| \in L_{2 \pi}^{1}$. Now, by Smirnov's theorem, both $U / H$ and $H W$ are in $\mathcal{H}_{\mathbb{C}}^{1}$ and therefore $(H W, U / H) \in \mathcal{P}(1-2 \lambda w)$ and $U^{*} W^{*}=z \in \mathcal{Z}(1-2 \lambda w)$. However $U^{*} W^{*}=(1-2 \lambda w)\left|W^{*}\right|^{2}$. To complete the proof it remains to establish that $w$ is realanalytic on $\Gamma, \Gamma \in \mathcal{G}$. Since $z \in \mathcal{Z}(1-2 \lambda w)$ is real-analytic on $\Gamma$, it follows from a bootstrap argument, exactly as in the local regularity theory in [15, Appendix], that $w$ is $C^{1, \alpha}$-smooth on $\Gamma$. The function $\varphi=\varphi_{\operatorname{Re}}+\mathrm{i} \varphi_{\operatorname{Im}}:=H W$ is analytic on $\left\{\mathrm{e}^{\mathrm{i} t} \mid t \in \Gamma\right\}$ (see Definition 1.6). Further,

$$
\begin{aligned}
w^{\prime}+\mathrm{i}\left(1+\mathcal{C} w^{\prime}\right) & =W^{*}=\frac{\varphi^{*}}{\mathcal{O}(\sqrt{|1-2 \lambda w|})} \\
& =\frac{\varphi^{*}}{\sqrt{|1-2 \lambda w|}} \exp \left(\mathrm{i} \mathcal{C}\left(\log \frac{1}{\sqrt{|1-2 \lambda w|}}\right)\right) .
\end{aligned}
$$

Let $v:=\log (\sqrt{|1-2 \lambda w|})^{-1}$ and $s:=\operatorname{sgn}\{1-2 \lambda w\}=$ const $\in\{ \pm 1\}$ on $\Gamma$. Then

$$
w=\frac{1}{2 \lambda}\left(1-\frac{s}{\mathrm{e}^{2 v}}\right) \text { and } \quad v^{\prime}=s \lambda \mathrm{e}^{3 v}\left(\varphi_{\mathrm{Re}} \cos \mathcal{C} v-\varphi_{\mathrm{Im}} \sin \mathcal{C} v\right)
$$

Let $u:=\mathcal{C} v$. Then

$$
v^{\prime}=\text { const } \mathrm{e}^{3 v}\left(\varphi_{\mathrm{Re}} \cos u-\varphi_{\operatorname{Im}} \sin u\right) .
$$

Since $w$ is $C^{1, \alpha}$-smooth on $\Gamma$, so is $v$, and hence $u$ (cf. [15, Appendix]). Now it follows from Lewy's theorem (see [8], [14, Section 4]) that $u$ and $v$ are real-analytic on $\Gamma$. Since $v \rightleftarrows w$ is a locally invertible analytic diffeomorphism, the analyticity of $w$ follows from that of $v$.

(d) The Riemann-Hilbert theory in [15] leads from Eq. (1.2) to equation

$$
(1-2 \lambda w)\left\{w^{\prime 2}+\left(1+\mathcal{C} w^{\prime}\right)^{2}\right\} \equiv 1,
$$

and in the process shows that

$$
W=\mathrm{i} / H, \quad \text { where } H(0)=1
$$

(cf. the remark at the beginning of Section 4.2). Since $H=\mathcal{O}(\sqrt{1-2 \lambda w})$,

$$
w^{\prime}+\mathrm{i}\left(1+\mathcal{C} w^{\prime}\right)=W^{*}=\frac{\mathrm{ie}^{-\mathrm{i} \mathcal{C} \log \sqrt{1-2 \lambda w}}}{\sqrt{1-2 \lambda w}} .
$$

So (1.8) implies $\mathcal{C} \log \sqrt{1-2 \lambda w}=\vartheta+\vartheta_{0}$, where $\vartheta_{0}$ is a measurable $2 \pi$-periodic function such that $\vartheta_{0}(t) \in 2 \pi \mathbb{Z}$ almost everywhere. Let us prove that $\vartheta_{0}$ is constant. Since $1 / \sqrt{1-2 \lambda w}=\left|W^{*}\right| \in L_{2 \pi}^{1}$ and $w$ is bounded, $\log \sqrt{1-2 \lambda w} \in L_{2 \pi}^{p}$ for any 
$p<\infty$. By Riesz's theorem $\vartheta+\vartheta_{0}$, and hence $\vartheta_{0}$, also belong to $L_{2 \pi}^{p}$ for any $p<\infty$. Now $|H(0)|=1$ implies that $\log \sqrt{1-2 \lambda w}$ has zero mean on $[-\pi, \pi]$ and therefore $\mathcal{C} \vartheta+\mathcal{C} \vartheta_{0}=-\log \sqrt{1-2 \lambda w}$. Consequently

$$
\mathrm{e}^{-\mathcal{C} \vartheta_{0}}=\sqrt{1-2 \lambda w} \mathrm{e}^{\mathcal{C} \vartheta} .
$$

It follows from [18, Vol. I, Chapter VII, Theorem (2.11)] that $\mathrm{e}^{\mathcal{C} \vartheta} \in L_{2 \pi}^{3}$. Therefore $\mathrm{e}^{-\mathcal{C} \vartheta_{0}} \in L_{2 \pi}^{3}$ and there exists a function $\Upsilon \in \mathcal{H}_{\mathbb{C}}^{3}$ such that $\Upsilon^{*}=\mathrm{e}^{\mathrm{i}\left(\vartheta_{0}+\mathrm{i} \mathcal{C} \vartheta_{0}\right)}$. Since $\vartheta_{0}(t) \in 2 \pi \mathbb{Z}$, we get $\Upsilon^{*} \geqslant 0$. Thus $\Upsilon$ is constant (see the proof of Lemma 1.4) and $\vartheta_{0}$ is also constant.

So $\mathcal{C} \vartheta=-\log \sqrt{1-2 \lambda w}$ and $\left|W^{*}\right|=\mathrm{e}^{\mathcal{C} \vartheta} \in L_{2 \pi}^{3}$. Finally, $w^{\prime} \in L_{2 \pi}^{3}$ and so $w$ is real analytic by part (a). This completes the proof.

Remark. - One can give a simpler proof of Theorem $1.7(\mathrm{~d})$ in the case $\vartheta_{1} \equiv 0$, i.e. when $\beta:=\|\vartheta\|_{\infty}<\pi / 6$. Indeed, let us consider the outer function $V=-\mathrm{i} W$. It follows from (1.8) that

$$
V^{*} \in \mathcal{A}_{\beta}:=\left\{\zeta=r \mathrm{e}^{\mathrm{i} \theta} \in \mathbb{C}:|\theta| \leqslant \beta, \quad r \geqslant 0\right\} .
$$

Since the angle $\mathcal{A}_{\beta}$ is convex and closed, the Poisson formula (see, e.g., [7, $1^{\circ}, \mathrm{B}$, Chapter II]) implies that $V(z) \in \mathcal{A}_{\beta},|z|<1$. Therefore $\operatorname{Re} V^{\frac{\pi}{2 \beta}}(z) \geqslant 0,|z|<1$ and hence $V^{\frac{\pi}{2 \beta}} \in \mathcal{H}_{\mathbb{C}}^{p}$ for any $p<1$ (see, e.g., [6, Exercise 13(a), Chapter II]). Since $\frac{\pi}{2 \beta}>3$, we have $V^{3} \in \mathcal{H}_{\mathbb{C}}^{1}$, i.e. $V \in \mathcal{H}_{\mathbb{C}}^{3}$, i.e. $w \in W_{2 \pi}^{1,3}$. Thus $w$ is real analytic by part (a).

\section{4. $\mathcal{P}(a)$ and $\mathcal{Z}(a)$}

The purpose of this section is to describe the set $\mathcal{P}(a)$, introduced in Definition 1.6, in the case when $a$ changes sign finitely often. (To describe $\mathcal{P}(a)$ in general is a more formidable task.) We then examine the special case of $a$ which changes sign twice.

\section{1. $a$ changes sign finitely often}

Changing the independent variable if necessary, suppose that $a(0)<0$ and that there exist points

$$
0<t_{1}<t_{2}<\cdots<t_{2 n-1}<t_{2 n}<2 \pi
$$

such that

$$
a(t) \geqslant 0 \quad \text { if } t \in\left[t_{2 k-1}, t_{2 k}\right], k=1, \ldots, n, \quad \text { and } \quad a(t)<0 \quad \text { otherwise. }
$$

We consider the problem (recall Definition 1.6)

$$
\psi^{*}=\Sigma(a) \overline{\varphi^{*}} \text { almost everywhere, } \quad \varphi, \psi \in \mathcal{H}_{\mathbb{C}}^{1} .
$$


Lemma 4.1 (Cf. [5, §41], [11, §78]). - Suppose that (4.1) and (4.2) hold. Then every solution of (4.3) is of the form

$$
(\varphi, \psi)=\left(\sum_{k=0}^{n} \bar{c}_{k} \varphi_{k}, \sum_{k=0}^{n} c_{k} \psi_{k}\right),
$$

where, for $k \in\{0, \ldots, n\}, c_{k}$ are complex numbers,

$$
\begin{array}{ll}
\psi_{0}(z)=X(z), & \varphi_{0}(z)=X(z) ; \\
\psi_{k}(z)=\frac{X(z)}{z-\mathrm{e}^{\mathrm{i} t_{2 k-1}},} & \varphi_{k}(z)=\frac{\mathrm{e}^{\mathrm{i} t_{2 k-1} z X(z)}}{\mathrm{e}^{\mathrm{i} t_{2 k-1}-z},}
\end{array}
$$

and

$$
X(z)=\mathrm{i} \prod_{k=1}^{n}\left(\frac{z \mathrm{e}^{-\frac{1}{2} \mathrm{i} t_{2 k-1}}-\mathrm{e}^{\frac{1}{2} \mathrm{i} t_{2 k-1}}}{z \mathrm{e}^{-\frac{1}{2} \mathrm{i} \mathrm{i}_{2 k}}-\mathrm{e}^{\frac{1}{2} \mathrm{i} t_{2 k}}}\right)^{1 / 2}, \quad z \in D .
$$

Here $\zeta^{1 / 2}$ is analytic in the complex plane cut along $(-\infty, 0]$ and is positive on $(0,+\infty)$. These solutions belong to $\mathcal{H}_{\mathbb{C}}^{q}, q<2$ and do not belong to $\mathcal{H}_{\mathbb{C}}^{2}$.

Proof. - It is not difficult to see that

$$
X_{k}(z)=\frac{z \mathrm{e}^{-\frac{1}{2} \mathrm{i} t_{2 k-1}}-\mathrm{e}^{\frac{1}{2} \mathrm{i} t_{2 k-1}}}{z \mathrm{e}^{-\frac{1}{2} \mathrm{i} t_{2 k}}-\mathrm{e}^{\frac{1}{2} \mathrm{i} t_{2 k}}}, \quad|z| \neq 1,
$$

is a conformal mapping of the open unit disk onto the lower complex half-plane, and of the complement of the closed unit disk onto the upper half-plane with one point removed. Moreover,

$$
X_{k}^{*}(t)=\frac{\sin \frac{t-t_{2 k-1}}{2}}{\sin \frac{t-t_{2 k}}{2}}
$$

In particular

$$
X_{k}^{*}(t)<0 \quad \text { for all } t \in\left(t_{2 k-1}, t_{2 k}\right), \quad X_{k}^{*}(t)>0 \quad \text { for all } t \in[0,2 \pi] \backslash\left[t_{2 k-1}, t_{2 k}\right] .
$$

Therefore $X_{k}^{1 / 2}$ is analytic in the complex plane cut along the closed $\operatorname{arc}\left\{\mathrm{e}^{\mathrm{i} t}: t_{2 k-1} \leqslant t \leqslant\right.$ $\left.t_{2 k}\right\}$ and

$$
\begin{aligned}
& \left(X_{k}^{1 / 2}\right)^{*}(t)>0 \text { for all } t \in[0,2 \pi] \backslash\left[t_{2 k-1}, t_{2 k}\right], \\
& \left(X_{k}^{1 / 2}\right)^{*}(t)=-\mathrm{i}\left|\left(X_{k}^{1 / 2}\right)^{*}(t)\right| \text { for all } t \in\left(t_{2 k-1}, t_{2 k}\right) .
\end{aligned}
$$

Since $X=\mathrm{i} \prod_{k=1}^{n} X_{k}^{1 / 2}$, we have

$$
\begin{aligned}
& X^{*}(t)=\mathrm{i}\left|X^{*}(t)\right| \quad \text { for all } t \in[0,2 \pi] \backslash \bigcup_{k=1}^{n}\left[t_{2 k-1}, t_{2 k}\right] \text { and } \\
& X^{*}(t)>0 \quad \text { for all } t \in \bigcup_{k=1}^{n}\left(t_{2 k-1}, t_{2 k}\right) .
\end{aligned}
$$


Hence

$$
X^{*} \overline{\left(X^{-1}\right)^{*}}=\Sigma(a)
$$

Let

$$
F:=\frac{\psi}{X}, \quad G:=\frac{\varphi}{X} .
$$

Then (4.3) and (4.7) imply that

$$
F^{*}=\overline{G^{*}} .
$$

By Hölder's inequality, $F, G \in \mathcal{H}_{\mathbb{C}}^{\tau}, \tau<2 / 3$. It follows from Smirnov's theorem that $F(z) \prod_{k=1}^{n}\left(z-\mathrm{e}^{\mathrm{i} t_{2 k-1}}\right)$ defines a function in $\mathcal{H}_{\mathbb{C}}^{1}$. Let the coefficients $\beta_{1}, \ldots, \beta_{n} \in \mathbb{C}$ be such that

$$
G_{0}(z):=G(z) \prod_{k=1}^{n}\left(z^{-1}-\mathrm{e}^{-\mathrm{i} t_{2 k-1}}\right)-\sum_{j=1}^{n} \beta_{j} z^{-j}
$$

is holomorphic in the open unit disk and put

$$
F_{0}(z):=F(z) \prod_{k=1}^{n}\left(z-\mathrm{e}^{\mathrm{i} t_{2 k-1}}\right)-\sum_{j=1}^{n} \overline{\beta_{j}} z^{j} .
$$

It is easy to see that $F_{0} \in \mathcal{H}_{\mathbb{C}}^{1}$ and $F_{0}^{*}=\overline{G_{0}^{*}}$ (by (4.9)). From Smirnov's and Carleman's theorems it follows that $G_{0} \equiv \beta_{0}, F_{0} \equiv \overline{\beta_{0}}$ for some $\beta_{0} \in \mathbb{C}$. Therefore

$$
F(z)=\frac{\sum_{j=0}^{n} \overline{\beta_{j}} z^{j}}{\prod_{k=1}^{n}\left(z-\mathrm{e}^{\left.\mathrm{i} t_{2 k-1}\right)}\right.}=c_{0}+\sum_{k=1}^{n} \frac{c_{k}}{z-\mathrm{e}^{\mathrm{i} t_{2 k-1}}}
$$

for some constants $c_{0}, c_{k} \in \mathbb{C}$. Define $F(z)$ for $|z|>1$ by (4.10). Since

$$
G(z)=\frac{\sum_{j=0}^{n} \beta_{j} z^{-j}}{\prod_{k=1}^{n}\left(z^{-1}-\mathrm{e}^{\left.-\mathrm{i} t_{2 k-1}\right)}\right.},
$$

we have

$$
G(z)=\overline{F(1 / \bar{z})}=\overline{c_{0}}+\sum_{k=1}^{n} \overline{c_{k}} \frac{\mathrm{e}^{\mathrm{i} t_{2 k-1} z}}{\mathrm{e}^{\mathrm{i} 2_{2 k-1}}-z},
$$

and the result follows from (4.8).

Lemma 4.2. - Suppose (4.1) and (4.2) hold and $a \in C_{2 \pi}^{\alpha}$. If $1 \leqslant p<\frac{2}{1+\alpha}$ then every solution $(\Phi, \Psi)$ of $(2.1)$ is given by

$$
\begin{aligned}
& (\Phi, \Psi)=\left(\sum_{k=0}^{n} \bar{\alpha}_{k} f_{k}, \sum_{k=0}^{n} \alpha_{k} g_{k}\right), \text { where } f_{k}:=\varphi_{k} / H, g_{k}=H \psi_{k}, \\
& k=0, \ldots, n, H=\mathcal{O}(\sqrt{|a|}) .
\end{aligned}
$$


Proof. - Suppose (2.1) has a non-trivial solution $(\Phi, \Psi) \in \mathcal{H}_{\mathbb{C}}^{p} \times \mathcal{H}_{\mathbb{C}}^{p}$. Let $\varphi:=\Phi H$, $\psi:=\Psi / H$. Then $\varphi, \psi \in \mathcal{H}_{\mathbb{C}}^{p}$ (cf. the proof of Corollary 2.3). Since $\varphi, \psi$ satisfy (4.3), they are given by linear combinations of $\varphi_{k}, \psi_{k}$, as in the preceding lemma. Therefore $\Phi, \Psi$ are given by linear combinations of $f_{k}$ and $g_{k}$, with complex coefficients $\alpha_{k}$, as in (4.11).

Remark. - Although this result does not assert the existence of solutions of Eq. (2.1) when $1 \leqslant p<2 /(1+\alpha)$, there are many cases where such solutions exist and consequently, in the light of Theorem 2.2, the result is sharp. To see this, suppose that, in addition to satisfying (4.1) and (4.2), $a \in C_{2 \pi}^{\alpha}, \alpha \in(0,1]$, has the property that $|a|^{-1} \in L_{2 \pi}^{q}$ for all $q<\alpha^{-1}$. Then $H \in \mathcal{H}_{\mathbb{C}}^{\infty}$ and $H^{-1} \in \mathcal{H}_{\mathbb{C}}^{2 q}$, for all $q<\alpha^{-1}$. Therefore

$$
\Psi:=H X \in \mathcal{H}_{\mathbb{C}}^{p} \quad \text { and } \quad \Phi:=X / H \in \mathcal{H}_{\mathbb{C}}^{p},
$$

for all $p \in[1,2 /(1+\alpha))$. Since $(\Phi, \Psi)$ is a non-trivial solution of (2.1) for any such choice of $a$, the result of Lemma 4.2 is optimal.

\section{2. $1-2 \lambda w$ changes sign twice}

In the water-wave problem (1.6)

$$
a:=(1-2 \lambda w) \quad \text { and } \quad U^{*}=a \overline{W^{*}} .
$$

We have seen that if $a$ changes sign only finitely many times then every solution of (1.6) is given by $W=\sum \bar{\alpha}_{k} f_{k}$ and $U=\sum \alpha_{k} g_{k}, \alpha_{k} \in \mathbb{C}$. By definition, $H(0) \in(0, \infty)$ is real, $f_{k}(0)=0, k=1, \ldots, n$, and

$$
X(0)=\mathrm{ie}^{-\mathrm{i} L / 4} \quad \text { where } \sum_{k=1}^{n}\left(t_{2 k}-t_{2 k-1}\right)=: L \in(0,2 \pi) .
$$

We will use the following results from [13, Chapter 17, Theorem 17 and Exercise 19].

(i) $G \in \mathcal{H}_{\mathbb{C}}^{1}$ is an outer function $(G=c \mathcal{O}(g)$ for some $c \in \mathbb{C}$ with $|c|=1$ and $g$ with $\left.\log |g| \in L_{2 \pi}^{1}\right)$ if and only if $|G(0)|=\mathcal{O}\left(G^{*}\right)(0)$.

(ii) If $G \in \mathcal{H}_{\mathbb{C}}^{1}$ is such that $G^{-1} \in \mathcal{H}_{\mathbb{C}}^{1}$, then $G$ is an outer function.

Remark. - In the case when $1-2 \lambda w>0$ almost everywhere we have seen that $\left|W^{*}\right|^{-2}=1-2 \lambda w, 1 / W(z)=$ const $\mathcal{O}(\sqrt{1-2 \lambda w})(z)$ and therefore, by (ii), $W$ is an outer function. Hence, by (i), in this case $H(0)=\mathcal{O}(\sqrt{1-2 \lambda w})(0)=\mathcal{O}\left(\left(W^{*}\right)^{-1}\right)(0)=$ $|W(0)|^{-1}=1$ (see (3.1)).

Now we investigate the possible existence of a solution $w$ of (1.2) for which $a:=$ $1-2 \lambda w$ changes sign exactly twice. In the notation of the preceding section, and rotating the disc if necessary, let $0<t_{1}=\gamma<t_{2}=2 \pi-\gamma$ and $a>0$ in $(\gamma, 2 \pi-\gamma)$. (Think of $\gamma$ as being small, with $\gamma=0$ in the case of the Stokes wave of greatest height with a crest at $t=0$.) Accordingly, from (4.11), there exists $\alpha_{k} \in \mathbb{C}, k=0,1$ with

$$
U(z)=\left\{\alpha_{0}+\frac{\alpha_{1}}{z-\mathrm{e}^{\mathrm{i} \gamma}}\right\} X(z) H(z) \quad \text { and } \quad W(z)=\left\{\overline{\alpha_{0}}+\frac{\overline{\alpha_{1}} z \mathrm{e}^{\mathrm{i} \gamma}}{\mathrm{e}^{\mathrm{i} \gamma}-z}\right\} \frac{X(z)}{H(z)},
$$


where

$$
H(z)=\mathcal{O}(\sqrt{|a|})(z) \quad \text { and } \quad X(z)=\mathrm{i}\left(\frac{\mathrm{e}^{\frac{1}{2} \mathrm{i} \gamma}-z \mathrm{e}^{-\frac{1}{2} \mathrm{i} \gamma}}{z^{\frac{1}{2} \mathrm{i} \gamma}-\mathrm{e}^{-\frac{1}{2} \mathrm{i} \gamma}}\right)^{1 / 2} .
$$

Therefore

$$
\begin{aligned}
& -\mathrm{i}=U(0)=\left\{\alpha_{0}-\alpha_{1} \mathrm{e}^{-\mathrm{i} \gamma}\right\} H(0) X(0), \\
& \mathrm{i}=W(0)=\overline{\alpha_{0}} X(0) / H(0) \quad \text { and } \quad X(0)=\mathrm{e}^{\frac{1}{2} \mathrm{i} \gamma}
\end{aligned}
$$

(see (3.1)). Let $h=H(0)$. (Recall that $h=1$ when $a>0$ almost everywhere.) Then

$$
\alpha_{0}=-\mathrm{i} h \mathrm{e}^{\frac{1}{2} \mathrm{i} \gamma} \quad \text { and } \quad \alpha_{1}=\frac{\mathrm{ie}^{\frac{1}{2} \mathrm{i} \gamma}}{h}-\mathrm{i} h \mathrm{e}^{3 \mathrm{i} \gamma / 2} .
$$

Therefore

$$
U(z)=\frac{\mathrm{ie}^{\frac{\mathrm{i} \gamma}{2}} X(z) H(z)}{z-\mathrm{e}^{\mathrm{i} \gamma}}\left(\frac{1}{h}-h z\right) \text { and } \quad W(z)=\left\{\frac{\mathrm{ie}^{\frac{\mathrm{i} \gamma}{2}} X(z)}{H(z)}\right\} \frac{h-\frac{z}{h}}{\mathrm{e}^{\mathrm{i} \gamma}-z} .
$$

Remark. $-W(z)=0$ if and only if $z=h^{2}$. Therefore the primitive, $F$, of $W\left(F^{\prime}=W\right)$ is a locally conformal mapping of the disc if and only if $h^{2} \geqslant 1$.

It now follows that

$$
U(z) W(z)=\frac{\left(h^{2} z-1\right)\left(\frac{z}{h^{2}}-1\right)}{\left(z-\mathrm{e}^{\mathrm{i} \gamma}\right)\left(z-\mathrm{e}^{-\mathrm{i} \gamma}\right)}
$$

and hence

$$
a\left|W^{*}(t)\right|^{2}=\frac{\frac{1}{2}\left(h^{2}+\frac{1}{h^{2}}\right)-\cos t}{\cos \gamma-\cos t}=: z(t), \quad z \in \mathcal{Z}(1-2 \lambda w), \quad \text { say. }
$$

Recall that if $a>0$ almost everywhere then

$$
U^{*} W^{*}=a\left|W^{*}(t)\right|^{2} \equiv 1
$$

We have already noted that, for the Stokes highest wave, $\gamma=0, a(0)=0$ and $h=1$. In general

$$
\begin{aligned}
& \frac{1}{2}\left(h^{2}+\frac{1}{h^{2}}\right) \geqslant 1 \quad \text { with equality if and only if } h^{2}=1, \\
& \cos \gamma \leqslant 1 \quad \text { with equality if and only if } \gamma=0 .
\end{aligned}
$$

In the light of linear theory in Section 2 , we can assert only that solution $w \in W_{2 \pi}^{1, p}$, $1 \leqslant p<3 / 2$, of (1.2) which changes sign exactly twice must also satisfy a generalised Bernoulli boundary condition for $w$ involving, as an additional parameter, the point $\gamma \in(0, \pi)$, where $1-2 \lambda w$ is zero at $\gamma$ and $2 \pi-\gamma$ : 


$$
\begin{gathered}
\left\{w^{\prime}(t)^{2}+\left(1+\mathcal{C} w^{\prime}(t)\right)^{2}\right\}=\frac{\frac{1}{2}\left(h^{2}+\frac{1}{h^{2}}\right)-\cos t}{(1-2 \lambda w(t))(\cos \gamma-\cos t)}, \\
\text { where } h=\exp \left(\frac{1}{4 \pi} \int_{-\pi}^{\pi} \log |1-2 \lambda w(s)| \mathrm{d} s\right) .
\end{gathered}
$$

As in (1.9), this equation corresponds to two free-boundary conditions, which relate the real and imaginary parts of a complex analytic function $\varphi+\mathrm{i} \psi$ in the (dimensionless) physical domain,

$$
\frac{1}{2} \frac{\frac{1}{2}\left(h^{2}+\frac{1}{h^{2}}\right)-\cos \varphi(x, y)}{\cos \gamma-\cos \varphi(x, y)}|\nabla \varphi(x, y)|^{2}+\lambda y \equiv \frac{1}{2}, \quad \psi(x, y) \equiv 0
$$

on the unknown free surface in the $(x, y)$-plane. But the pressure on the free-surface is not constant unless $\gamma=0$ and $h=1$. It remains a possibility that the nonlinear waterwave problem (1.2) yields this additional information, but a proof has so far eluded us. Therefore although the heuristic arguments which led to the variational formulation of the Stokes-wave problem based on the functional $\mathcal{J}$ suggest that it should be so, we are unable to assert that solutions of (1.2) in the Hardy space $\mathcal{H}_{\mathbb{R}}^{1,1}$, or even in the Sobolev space $W_{2 \pi}^{1, p}, 1<p<3 / 2$, give rise to Stokes waves.

\section{Acknowledgement}

J.F. Toland was supported by an EPSRC Senior Fellowship.

\section{REFERENCES}

[1] K.I. Babenko, Some remarks on the theory of surface waves of finite amplitude, Soviet Math. Dokl. 35 (3) (1987) 599-603; See also loc. cit. 647-650.

[2] B. Buffoni, E.N. Dancer, J.F. Toland, The regularity and local bifurcation of Stokes waves, Arch. Rational Mech. Anal. 152 (3) (2000) 207-240.

[3] B. Buffoni, E.N. Dancer, J.F. Toland, The sub-harmonic bifurcation of Stokes waves, Arch. Rational Mech. Anal. 152 (3) (2000) 241-270.

[4] A.I. Dyachenko, E.A. Kuznetsov, M.D. Spector, V.E. Zakharov, Analytic description of the free surface dynamics of an ideal fluid (canonical formalism and conformal mapping), Phys. Lett. A 1 (1996) 73-79.

[5] F.D. Gakhov, Boundary Value Problems, Pergamon Press, Oxford, 1966.

[6] J.B. Garnett, Bounded Analytic Functions, Academic Press, New York, 1981.

[7] P. Koosis, Introduction to $H_{p}$ Spaces, 2nd Edition, Cambridge University Press, Cambridge, 1999.

[8] H. Lewy, A note on harmonic functions and a hydrodynamic application, Proc. Amer. Math. Soc. 3 (1952) 111-113.

[9] E.H. Lieb, M. Loss, Analysis, in: Graduate Studies in Mathematics, Vol. 14, American Mathematical Society, Providence, RI, 1997.

[10] J.B. McLeod, The Stokes and Krasovskii conjectures for the wave of greatest height, in: Studies in Applied Math., Vol. 98, 1997, pp. 311-334. In pre-print-form: Univ. of Wisconsin Mathematics Research Center Report Number 2041, 1979 (sic). 
[11] N.I. Muskhelishvili, Singular Integral Equations, Wolters-Noordhoff Publishing, Groningen, 1972.

[12] P.I. Plotnikov, Non-uniqueness of solutions of the problem of solitary waves and bifurcation of critical points of smooth functionals, Math. USSR Izvestiya 38 (2) (1992) 333-357.

[13] W. Rudin, Real and Complex Analysis, 3rd Edition, McGraw-Hill, New York, 1986.

[14] J.F. Toland, Stokes waves, Topological Methods in Nonlinear Analysis 7 (1996) 1-48; Topological Methods in Nonlinear Analysis 8 (1997) 412-414.

[15] J.F. Toland, Regularity of Stokes waves in Hardy spaces and in spaces of distributions, J. Math. Pure Appl. 79 (9) (2000) 901-917.

[16] J.F. Toland, On a pseudo-differential equation for Stokes waves, Arch. Rational Mech. Anal. 162 (2002) 179-189.

[17] A. Torchinsky, Real-Variable Methods in Harmonic Analysis, Academic Press, Orlando, 1986.

[18] A. Zygmund, Trigonometric Series I \& II, Cambridge University Press, Cambridge, 1959. 